\title{
Packet-Level Error Models for Digital Wireless Channels
}

\author{
Cheng-Xiang Wang \\ Faculty of Engineering and Science \\ Agder University College \\ Grooseveien 36, NO-4876 Grimstad, Norway \\ Email: cheng.wang@hia.no
}

\author{
Wen $\mathrm{Xu}$ \\ Department of Mobile Phone Development \\ Siemens AG \\ Grillparzerstr. 10-18, D-81675 Munich, Germany \\ Email: wen.xu@siemens.com
}

\begin{abstract}
Packet-level error models have great significance for the design and performance evaluation of higher layer wireless communication protocols. These models aim to characterize the statistical behavior of bursty packet error sequences encountered in digital wireless channels. In this paper, target packet error sequences are generated by computer simulations of a coded general packet radio service (GPRS) system with a typical urban (TU) channel and co-channel interference. A general design procedure of a generative model is then proposed by using a properly parameterized and sampled deterministic process with a threshold detector and two parallel mappers. Simulation results indicate that the proposed deterministic process based generative model (DPBGM) allows us to approximate very well the characteristics of the target packet error sequences with respect to the gap distribution (GD), error-free run distribution (EFRD), error cluster distribution (ECD), error burst distribution (EBD), error-free burst distribution (EFBD), block error probability distribution (BEPD), and packet error correlation function (PECF). Importantly, it is shown that both the accuracy and the efficiency of the suggested DPBGM outperform those of a widely used Markov model.
\end{abstract}

Keywords - Error models, packet error sequences, burst error statistics, deterministic processes, wireless protocols.

\section{INTRODUCTION}

With the evolution of wireless networks, the complexity of communication protocols increases. For the design, optimization, and performance evaluation of higher layer wireless communication protocols, it is essential to have a proper understanding and an accurate modeling of network error events [1]. In particular, a packet or frame error process which describes the successes and failures of packet transmissions in digital wireless channels is of primary importance. Therefore, many researches have been performed for the development of packet-level error models for characterizing packet error sequences.

The description of the statistical behavior of bit or packet error sequences can be performed by means of both descriptive [2] and generative approaches [3]. A descriptive model describes the statistics of target error sequences obtained directly from experimental results. A generative model specifies a mechanism that generates error sequences with desired statistics [3]. Clearly, a generative model can greatly reduce the computational effort for generating long error sequences and therefore enables fast simulations.
Traditional bit-level or packet-level generative models are based on Markov chains [3]-[6] or hidden Markov chains [8][11]. Especially, much attention was dedicated to simplified Fritchman's models (SFMs) [4], which belong to the finitestate Markov models with only one error state. Recently, other promising mechanisms, e.g., chaos equations [10], [12], stochastic context-free grammars [11], and sum-of-sinusoids based deterministic processes [13]-[17], were successfully applied to generate bit error sequences with good burst error statistics. In principle, these bit-level generative models [10][17] can further be extended to the simulation of packet error sequences by properly tuning the involved parameters. However, the accuracy of the resulting packet-level generative models needs to be validated. The aim of this paper is to extend the idea of [16] and develop a DPBGM for the simulation of packet error sequences. A GPRS digital wireless network at the radio link layer [18] is adoped to provide target packet error sequences. Simulation results show that the proposed DPBGM enables us to approximate closely all the desired burst error statistics of the underlying descriptive model.

The paper is organized as follows. Section II introduces the definitions of the terms and interested burst error statistics related to binary packet error sequences. A general design procedure of the proposed DPBGM is addressed in Section III. Section IV presents the adopted GPRS system and the resulting packet error sequences. In this section, the burst error statistics of the underlying descriptive model, the proposed generative model, and a SFM are also compared. Finally, the conclusions are drawn in Section V.

\section{BURST ERRor Statistics}

For reasons of clarity, let us first introduce the terms and relevant burst error statistics we use in this paper to characterize binary packet error sequences. Of course, the definitions of the terms were chosen in such a way that they are convenient for the development of a generative model based on deterministic processes.

A packet error sequence is represented here by a binary sequence of ones and zeros, where a " 1 " denotes an error packet, while a " 0 " denotes a correct packet. In consistent with [16], a gap is defined as a string of consecutive zeros between two ones, having a length equal to the number of zeros. An 
error cluster is a region where the errors occur consecutively and has a length equal to the number of ones [4]. An errorfree burst is defined as an all-zero sequence with the number of zeros equal to or larger than $\eta$, which is a positive integer [5]. An error burst is a sequence of zeros and ones starting and ending with a one, and separated from neighboring error bursts by error-free bursts [5]. This implies that the minimum length of an error burst is 1 and the number of consecutive zeros within an error burst is less than $\eta$.

The above term definitions allow further observations: an error sequence is the combination of consecutive error bursts and error-free bursts, while error bursts can further be subdivided into gaps and error clusters. Consequently, an error sequence can concisely be represented by listing the successive error burst lengths and error-free burst lengths. It follows that an error burst recorder $\mathbf{E B}_{r e c}$ and an error-free burst recorder $\mathbf{E F B}_{r e c}$ are obtained. Here, $\mathbf{E B}_{r e c}$ is a vector which counts successive error burst lengths, while $\mathbf{E F B}_{r e c}$ records successive error-free burst lengths. Let us denote the minimum value as $m_{B 1}$ and the maximum value as $m_{B 2}$ in $\mathbf{E B}_{r e c}$. This means that the lengths $m_{e}$ of error bursts satisfy $m_{B 1} \leq m_{e} \leq$ $m_{B 2}$. By analogy, the minimum value and the maximum value in $\mathbf{E F B}_{\text {rec }}$ are denoted as $m_{\bar{B} 1}$ and $m_{\bar{B} 2}$, respectively. For the convenience of developing the DPBGM in Section III, the following quantities are necessary to further be defined:

1) $N_{t}$ : the total length of the target packet error sequence.

2) $\mathcal{N}_{E B}$ : the total number of error bursts, which equals the number of entries in $\mathbf{E B}_{\text {rec }}$.

3) $\mathcal{N}_{E F B}$ : the total number of error-free bursts, which equals the number of entries in $\mathbf{E F B}_{\text {rec }}$.

4) $N_{E B}\left(m_{e}\right)$ : the number of error bursts of length $m_{e}$ in $\mathbf{E B}_{\text {rec }}$. Apparently, $\sum_{m_{e}=m_{B 1}}^{m_{B 2}} N_{E B}\left(m_{e}\right)=\mathcal{N}_{E B}$ holds.

5) $N_{E F B}\left(m_{\bar{e}}\right)$ : the number of error-free bursts of length $m_{\bar{e}}$ in $\mathbf{E F B}_{r e c}$. Similarly, $\sum_{m_{\bar{e}}=m_{\bar{B} 1}}^{m_{\bar{B} 2}} N_{E F B}\left(m_{\bar{e}}\right)=\mathcal{N}_{E F B}$ holds.

6) $\mathcal{R}_{B}$ : the ratio of the mean value $M_{E B}$ of error bursts to the mean value $M_{E F B}$ of error-free bursts, i.e., $\mathcal{R}_{B}=$ $M_{E B} / M_{E F B}$.

7) $\mathbf{E C G}_{i}$ : a vector which lists successive error cluster lengths and gap lengths corresponding to each entry of $\mathbf{E B}_{r e c}$. Clearly, $i=1,2, \ldots, \mathcal{N}_{E B}$. Note that each vector $\mathbf{E C G}_{i}$ has an odd number of entries, with error cluster lengths as odd entries and gap lengths as even entries.

In relevant to binary packet error sequences, we are interested in the following burst error statistics [16]:

1) $G\left(m_{g}\right)$ : the GD, which is defined as the cumulative distribution function (CDF) of gap lengths $m_{g}$.

2) $P\left(0^{m_{0}} / 1\right)$ : the EFRD, which is the probability that a one is followed by at least $m_{0}$ zeros [4]. From the given $\mathrm{GD}$, the EFRD can be calculated and vice versa. Clearly, $P\left(0^{m_{0}} / 1\right)$ is a monotonically decreasing function of $m_{0}$ such that $P\left(0^{0} / 1\right)=1$ and $P\left(0^{m_{0}} / 1\right) \rightarrow 0$ as $m_{0} \rightarrow \infty$.

3) $P\left(1^{m_{c}} / 0\right)$ : the ECD, which is the probability that a zero is followed by $m_{c}$ or more ones [4].

4) $P_{E B}\left(m_{e}\right)$ : the EBD, which is the CDF of error burst lengths $m_{e}$.

5) $P_{E F B}\left(m_{\bar{e}}\right)$ : the EFBD, which is the CDF of error-free burst lengths $m_{\bar{e}}$.

6) $P(m, n)$ : the BEPD, which is the probability that at least $m$ out of $n$ packets are in error.

7) $\rho(\Delta k)$ : the PECF, which is the conditional probability that the $\Delta k$ th packet following an error packet is also in error.

It is important to mention that these burst error statistics will later be used as the performance criteria for the evaluation of the developed generative models in Section IV. In general, one generative model is preferred to another if it better fits the important statistics, e.g., the BEPD and PECF, of the underlying descriptive model.

\section{The Generative Model}

It is widely accepted that the second order statistics of fading envelope processes are closely related to the statistics of burst errors. This suggests the possibility that fading processes can be used as an error generation mechanism. Inspired by the promising advantages of deterministic sum-of-sinusoids channel simulators [19], [20], in this section we will develop a general design procedure of generating packet error sequences based on deterministic processes.

The generation of error bursts and error-free bursts is naturally related to fading intervals and inter-fade intervals of a fading process, respectively. The key idea behind the proposed generative model is to derive directly from a deterministic process an error burst length generator and an error-free burst length generator. The employed deterministic process $\tilde{\zeta}(t)$ must be properly parameterized and sampled with a certain sampling interval $T_{A}$. A threshold detector then follows after the sampled deterministic process $\tilde{\zeta}\left(k T_{A}\right)$, where $k$ is a nonnegative integer. During the simulation, the level of the deterministic process will vary and cross the given threshold $r_{t h}$ from time to time. Error-free bursts are supposed to be produced at the model's output if the level of $\tilde{\zeta}\left(k T_{A}\right)$ is above $r_{t h}$. The lengths of the generated error-free bursts equal the numbers of samples in the corresponding inter-fade intervals of $\tilde{\zeta}\left(k T_{A}\right)$. On the other hand, when the level of $\tilde{\zeta}\left(k T_{A}\right)$ falls below $r_{t h}$, then error bursts will occur. The error burst lengths equal the numbers of samples in the corresponding fading intervals of $\tilde{\zeta}\left(k T_{A}\right)$. Consequently, an error burst length generator $\widetilde{\mathbf{E B}}_{r e c}$ and an error-free burst length generator $\widetilde{\mathbf{E F B}}_{r e c}$ are obtained. Similar to the notations used for the descriptive model in Section II, we simply put the tilde sign on all affected symbols for the generative model. For example, we write $\tilde{m}_{B 1}, \tilde{\mathcal{N}}_{E F B}$, and $\tilde{N}_{E B}\left(m_{e}\right)$.

\section{A. The Parametrization of the Sampled Deterministic Process}

The first step for the design of the proposed generative model lies in the parametrization of the employed deterministic process based on the known quantities obtained from the target packet error sequences. In the following, a general idea is described to estimate the parameters of the underlying 
deterministic process used in the generative model. The levelcrossing rate (LCR) $\tilde{N}_{\zeta}\left(r_{t h}\right)$ at the chosen threshold $r_{t h}$ is fitted to the desired occurrence rate $R_{E B}=N_{p} \mathcal{N}_{E B} / T_{t}$ of error bursts. Here, $N_{p}$ stands for the packet size and $T_{t}$ denotes the total transmission time of the reference transmission system, from which the target packet error sequence of length $N_{t}$ is obtained. Also, the ratio $\tilde{\mathcal{R}}_{B}$ of the average duration of fades (ADF) $\tilde{T}_{\zeta_{-}}\left(r_{t h}\right)$ at $r_{t h}$ to the average duration of inter-fades (ADIF) $\tilde{T}_{\zeta_{+}}\left(r_{t h}\right)$ at $r_{t h}$ is adapted to the desired ratio $\mathcal{R}_{B}=M_{E B} / M_{E F B}$. Moreover, we must ensure that the sampling interval $T_{A}$ is chosen sufficiently small in order to detect most of the level crossings and fading intervals at deep levels, i.e., $r_{t h} \ll 1$.

Basically, any forms of deterministic processes, e.g., in [13]-[17] and [19]-[21], with different degrees of complexities can be utilized for our purpose. In this paper, we will only consider the following simple continuous-time deterministic process [16]

$$
\tilde{\zeta}(t)=\left|\tilde{\mu}_{1}(t)+j \tilde{\mu}_{2}(t)\right|
$$

where

$$
\tilde{\mu}_{i}(t)=\sum_{n=1}^{N_{i}} c_{i, n} \cos \left(2 \pi f_{i, n} t+\theta_{i, n}\right), \quad i=1,2 .
$$

In (2), $N_{i}$ defines the number of sinusoids, $c_{i, n}, f_{i, n}$, and $\theta_{i, n}$ are called the gains, the discrete frequencies, and the phases, respectively. By using the method of exact Doppler spread (MEDS) [20], the phases $\theta_{i, n}$ are considered as the realizations of a random generator uniformly distributed over $(0,2 \pi]$, while $c_{i, n}$ and $f_{i, n}$ are given by

$$
\begin{gathered}
c_{i, n}=\sigma_{0} \sqrt{\frac{2}{N_{i}}} \\
f_{i, n}=f_{\max } \sin \left[\frac{\pi}{2 N_{i}}\left(n-\frac{1}{2}\right)\right]
\end{gathered}
$$

respectively. Here, $\sigma_{0}$ is the square root of the mean power of $\tilde{\mu}_{i}(t)$ and $f_{\max }$ represents the maximum Doppler frequency.

When using the MEDS with $N_{i} \geq 7$, it has been shown in [20] that the LCR $\tilde{N}_{\zeta}(r)$ of $\tilde{\zeta}(t)$ is very close to the LCR $N_{\zeta}(r)$ of a Rayleigh process, which is given by

$$
N_{\zeta}(r)=\sqrt{\frac{\beta}{2 \pi}} p_{\zeta}(r), \quad r \geq 0
$$

where

$$
\beta=2\left(\pi \sigma_{0} f_{\max }\right)^{2}
$$

and

$$
p_{\zeta}(r)=\frac{r}{\sigma_{0}^{2}} \exp \left(-\frac{r^{2}}{2 \sigma_{0}^{2}}\right), r \geq 0
$$

denotes the Rayleigh distribution. It can also be shown that the ADF $\tilde{T}_{\zeta_{-}}(r)$ and the ADIF $\tilde{T}_{\zeta_{+}}(r)$ of $\tilde{\zeta}(t)$ approximate very well the desired quantities $T_{\zeta_{-}}(r)$ and $T_{\zeta_{+}}(r)$, respectively, of a Rayleigh process. They are given by

$$
T_{\zeta_{-}}(r)=\sqrt{\frac{2 \pi}{\beta}} \frac{\sigma_{0}^{2}}{r}\left[\exp \left(\frac{r^{2}}{2 \sigma_{0}^{2}}\right)-1\right], r \geq 0
$$

$$
T_{\zeta_{+}}(r)=\sqrt{\frac{2 \pi}{\beta}} \frac{\sigma_{0}^{2}}{r}, r \geq 0 .
$$

It then follows that the ratio $\tilde{\mathcal{R}}_{B}$ can be expressed as

$$
\tilde{\mathcal{R}}_{B}=\frac{\tilde{T}_{\zeta_{-}}\left(r_{t h}\right)}{\tilde{T}_{\zeta_{+}}\left(r_{t h}\right)} \approx \frac{T_{\zeta_{-}}\left(r_{t h}\right)}{T_{\zeta_{+}}\left(r_{t h}\right)}=\exp \left(\frac{r_{t h}^{2}}{2 \sigma_{0}^{2}}\right)-1 .
$$

The confronted task now is to find a proper parameter vector $\boldsymbol{\Psi}=\left(N_{1}, N_{2}, r_{t h}, \sigma_{0}, f_{\max }, T_{A}\right)$, which influences the statistical behavior of the underlying deterministic process, so that the following conditions can be fulfilled: $\mathcal{R}_{B}=\frac{T_{\zeta_{-}}\left(r_{t h}\right)}{T_{\zeta_{+}}\left(r_{t h}\right)}$ and $R_{E B}=N_{\zeta}\left(r_{t h}\right)$. For our purpose at hand, it is not necessary to include all elements of the parameter vector $\Psi$ in the design. We can first fix $N_{1}, N_{2}$, and $r_{t h}$ by choosing reasonable values, e.g., $N_{1}=9, N_{2}=10$, and $r_{t h}=0.09$. Then, performing $\mathcal{R}_{B}=\frac{T_{\zeta_{-}}\left(r_{t h}\right)}{T_{\zeta_{+}}\left(r_{t h}\right)}, \sigma_{0}$ can be calculated according to the following expression

$$
\sigma_{0}=\frac{r_{t h}}{\sqrt{2 \ln \left(1+\mathcal{R}_{B}\right)}} .
$$

With the help of the relation $R_{E B}=N_{\zeta}\left(r_{t h}\right), f_{\max }$ is given by

$$
f_{\max }=\frac{N_{p} \mathcal{N}_{E B}}{\sqrt{\pi} \sigma_{0} T_{t} p_{\zeta}\left(r_{t h}\right)} .
$$

The substitution of (7) into (12) yields the following explicit expression

$$
f_{\text {max }}=\frac{N_{p} \mathcal{N}_{E B}\left(1+\mathcal{R}_{B}\right)}{T_{t} \sqrt{2 \pi \ln \left(1+\mathcal{R}_{B}\right)}} .
$$

Equation (13) clearly states that $f_{\max }$ is completely determined by $N_{p}, \mathcal{N}_{E B}, \mathcal{R}_{B}$, and $T_{t}$, but not influenced by $r_{t h}$ and $\sigma_{0}$. The sampling interval $T_{A}$ for small values of $r_{t h}$ can suitably be chosen as follows [16]

$$
T_{A} \approx \frac{4}{\sqrt{5 \pi}} T_{\zeta_{-}}\left(r_{t h}\right) \sqrt{-1+\sqrt{1+10 q_{s} / 3}}
$$

where $q_{s}$ is a very small quantity determining the maximum measurement error of the LCR. This implies that the probability of undetectable level crossings at $r_{t h}$ is not larger than $q_{s}$. Using (8), (14) can finally be expressed as

$$
T_{A} \approx \frac{4 \sigma_{0}\left[\exp \left(\frac{r_{t h}^{2}}{2 \sigma_{0}^{2}}\right)-1\right]}{\sqrt{5} \pi r_{t h} f_{\max }} \sqrt{-1+\sqrt{1+10 q_{s} / 3}} .
$$

With the resulting parameter vector $\boldsymbol{\Psi}$, a sampled deterministic process $\tilde{\zeta}\left(k T_{A}\right)$ is simulated within the necessary time interval $\left[0, \tilde{T}_{t}\right]$, i.e., $0 \leq k T_{A} \leq \tilde{T}_{t}$. Here, $\tilde{T}_{t}=T_{t} \tilde{N}_{t} / N_{t}$ with $\tilde{N}_{t}$ denoting the required length of the generated packet error sequence. The total numbers of the generated error busts $\tilde{\mathcal{N}}_{E B}$ and error-free bursts $\tilde{\mathcal{N}}_{E F B}$ can approximately be estimated from $\tilde{\mathcal{N}}_{E B}=\left\lfloor\frac{\tilde{N}_{t}}{N_{t}} \mathcal{N}_{E B}\right\rfloor$ and $\tilde{\mathcal{N}}_{E F B}=\left\lfloor\frac{\tilde{N}_{t}}{N_{t}} \mathcal{N}_{E F B}\right\rfloor$, respectively. Here, $\lfloor x\rfloor$ stands for the nearest integer to $x$ towards minus infinity. In this manner, an error burst length recorder $\widetilde{\mathbf{E B}}_{r e c}$ with $\tilde{\mathcal{N}}_{E B}$ entries and an error-free burst length recorder $\widetilde{\mathbf{E F B}}_{r e c}$ with $\tilde{\mathcal{N}}_{E F B}$ entries are derived. 


\section{B. The Mappers}

Detailed investigations have shown that the obtained recorders $\widetilde{\mathbf{E B}}_{r e c}$ and $\widetilde{\mathbf{E F B}}_{r e c}$ are not suitable to directly generate an acceptable EBD and EFBD, respectively. Therefore, the second step of the design procedure is to develop two appropriate mappers, which map the lengths of the generated error bursts and error-free bursts to the corresponding desired lengths. The idea of the mappers is to properly modify $\widetilde{\mathbf{E B}}_{r e c}$ and $\widetilde{\mathbf{E F B}}_{\text {rec }}$ such that $\tilde{N}_{E B}\left(m_{e}\right)=$ $N_{E B}^{\prime}\left(m_{e}\right)$ and $\tilde{N}_{E F B}\left(m_{\bar{e}}\right)=N_{E F B}^{\prime}\left(m_{\bar{e}}\right)$ hold, respectively. Here, $N_{E B}^{\prime}\left(m_{e}\right)$ equals $\left\lfloor\frac{N_{t}}{N_{t}} N_{E B}\left(m_{e}\right)\right\rfloor$ or $\left\lfloor\frac{\tilde{N}_{t}}{N_{t}} N_{E B}\left(m_{e}\right)\right\rfloor+$ 1 for different error burst lengths $m_{e}$ in order to fulfill $\sum_{m_{e}=m_{B 1}}^{m_{B 2}} N_{E B}^{\prime}\left(m_{e}\right)=\tilde{\mathcal{N}}_{E B}$. Similarly, $N_{E F B}^{\prime}\left(m_{\bar{e}}\right)$ equals $\left\lfloor\frac{\tilde{N}_{t}}{N_{t}} N_{E F B}\left(m_{\bar{e}}\right)\right\rfloor$ or $\left\lfloor\frac{\tilde{N}_{t}}{N_{t}} N_{E F B}\left(m_{\bar{e}}\right)\right\rfloor+1$ for different errorfree burst lengths $m_{\bar{e}}$ to satisfy $\sum_{\tilde{P}_{\bar{e}}=m_{\bar{B} 1}}^{m_{\bar{B} 2}} N_{E F B}^{\prime}\left(m_{\bar{e}}\right)=$ $\tilde{\mathcal{N}}_{E F B}$. Note that the resulting EBD $\tilde{P}_{E B}\left(m_{e}\right)$ will match well the desired EBD $P_{E B}\left(m_{e}\right)$, since $\tilde{N}_{E B}\left(m_{e}\right)$ is almost proportional to $N_{E B}\left(m_{e}\right)$. Also, the resulting $\operatorname{EFBD} \tilde{P}_{E F B}\left(m_{\bar{e}}\right)$ will be close to the desired one $P_{E F B}\left(m_{\bar{e}}\right)$.

Next, we will only show how to design the mapper for $\widetilde{\mathbf{E B}}_{r e c}$. The same idea applies also to $\widetilde{\mathbf{E F B}}_{r e c}$. For each error burst length value $m_{e}\left(m_{B 1} \leq m_{e} \leq m_{B 2}\right)$, we first find the corresponding values $\ell_{m_{e}}^{1}$ and $\ell_{m_{e}}^{2}\left(\tilde{m}_{B 1} \leq \ell_{m_{e}}^{1}, \ell_{m_{e}}^{2} \leq \tilde{m}_{B 2}\right)$ in $\widetilde{\mathbf{E B}}_{r e c}$ to satisfy the following conditions

$$
\begin{aligned}
& \sum_{l=\ell_{m_{e}}^{1}}^{\ell_{m_{e}}^{2}-1} \tilde{N}_{E B}(l)<N_{E B}^{\prime}\left(m_{e}\right) \\
& \sum_{l=\ell_{m_{e}}^{1}}^{\ell_{m_{e}}^{2}} \tilde{N}_{E B}(l) \geq N_{E B}^{\prime}\left(m_{e}\right) .
\end{aligned}
$$

Let us define

$$
N_{\ell_{m_{e}}^{2}}=N_{E B}^{\prime}\left(m_{e}\right)-\sum_{l=\ell_{m_{e}}^{1}}^{\ell_{m_{e}}^{2}-1} \tilde{N}_{E B}(l) .
$$

Clearly, $\sum_{l=\ell_{m_{e}}^{1}}^{\ell_{m_{e}}^{2}-1} \tilde{N}_{E B}(l)+N_{\ell_{m_{e}}^{2}}=N_{E B}^{\prime}\left(m_{e}\right)$ holds. This indicates that if we map all error burst lengths between $\ell_{m_{e}}^{1}$ and $\ell_{m_{e}}^{2}-1$, while only $N_{\ell_{m_{e}}^{2}}$ error burst lengths of $\ell_{m_{e}}^{2}$ in $\widetilde{\mathbf{E B}}_{r e c}$ to $m_{e}$, then $\tilde{N}_{E B}\left(m_{e}\right)=N_{E B}^{\prime}\left(m_{e}\right)$ will be satisfied. Note that $\ell_{m_{B 1}}^{1}=\tilde{m}_{B 1}$ and $\ell_{m_{B 2}}^{2}=\tilde{m}_{B 2}$ hold. In summary, the mapper for the error burst length generator works as follows: if $l\left(\ell_{m_{e}}^{1} \leq l<\ell_{m_{e}}^{2}-1\right)$ samples of the deterministic process are observed in a fading interval, then a mapping $l \rightarrow m_{e}$ is first performed and afterwards an error burst with length $m_{e}$ is generated.

\section{The Generation of Packet Error Sequences}

The third step for the design procedure of the DPBGM is to generate packet error sequences from the modified recorders $\widetilde{\mathbf{E B}}_{r e c}$ and $\widetilde{\mathbf{E F B}}_{r e c}$ after the mappers. The generation of error-free bursts is straightforward since each entry of $\widetilde{\mathbf{E F B}}_{r e c}$ is simply interpreted as the number of consecutive zeros.
For generating error bursts, it is convenient to first construct parameter vectors $\widetilde{\mathbf{E C G}}_{j}\left(j=1,2, \ldots, \tilde{\mathcal{N}}_{E B}\right)$, which reflect the infrastructure of each error burst in $\widetilde{\mathbf{E B}}_{r e c}$ by listing the corresponding consecutive cluster lengths and gap lengths. To this end, we first have to find all vectors $\mathbf{E} \mathbf{C G}_{i}$ corresponding to error bursts with length $m_{e}$ in $\mathbf{E B}_{r e c}$. Then, for all error bursts with the same length $m_{e}$ in $\widetilde{\mathbf{E B}}_{r e c}$, we assign randomly $\mathbf{E C G}_{j}$ from all possible vectors $\mathbf{E C G}_{i}$. With such a vector $\mathbf{E C G}_{j}$, an error burst is generated by combining consecutive error clusters (ones) and gaps (zeros). The resulting packet error sequence is simply the combination of consecutively generated error bursts and error-free bursts. The block diagram of the obtained generative model is depicted in Fig. 1. It should be emphasized that, although the simulation set-up phase (determining the parameters and designing the mappers) of the proposed DPBGM requires relatively long time, the simulation run phase (generation of packet error sequences) is fast. This is due to the fact that the developed DPBGM avoids a bit-by-bit processing of error sequences by directly determining error burst and error-free burst lengths.

\section{Simulation Results and Discussions}

In this section, we validate the accuracy and efficiency of the proposed DPBGM by applying the mechanism to experimental binary packet error sequences collected at the radio link layer of a coded GPRS system. The transmitter part of the GPRS system consists of a convolutional encoder, a burst interleaver, and a Gaussian minimum shift keying (GMSK) modulator, while the receiver side includes a GMSK demodulator, a Viterbi equalizer, a burst deinterleaver, a convolutional decoder, and a cyclic redundancy check (CRC) for error detection. As an example, the coding scheme 3 (CS3) specified for GPRS [18] was employed by the convolutional encoder. Here, we have considered a TU propagation channel profile with co-channel interference. The mobile speed was set to be $v=3 \mathrm{~km} / \mathrm{h}$. No frequency hopping was used. The data were transmitted by frames or packets of $N_{p}=456$ bits with a transmission rate of $F_{s}=270.8 \mathrm{~kb} / \mathrm{s}$. One frame contains four time-division multiple access (TDMA) bursts of 114 bits. A packet error sequence is formed by assigning a zero to a correctly decoded packet and a one to an error packet, which contains at least one undecodable error. Fig. 2 plots the resulting average frame error rate (FER) of the coded GPRS system. The target packet error sequences of length $N_{t}=10^{6}$ were produced at carrier-to-interference ratios (CIRs) of $5 \mathrm{~dB}$, $7 \mathrm{~dB}, 8 \mathrm{~dB}, 9 \mathrm{~dB}, 11 \mathrm{~dB}, 13 \mathrm{~dB}, 15 \mathrm{~dB}, 17 \mathrm{~dB}, 19 \mathrm{~dB}, 21 \mathrm{~dB}$, $23 \mathrm{~dB}$, and $25 \mathrm{~dB}$. The total transmission time is therefore $T_{t}=456 N_{t} / F_{s} \approx 1684 \mathrm{~s}$, which is about 28 minutes.

Error sequences of length $\tilde{N}_{t}=1.2 \times 10^{6}$ were generated by using the proposed DPBGM. The GDs, the EFRDs, the ECDs, the EBDs and EFBDs with $\eta=80$, the BEPDs with $n=55$, and the PECFs calculated from the target and generated packet error sequences were compared. Also, the relevant results of a six-state SFM [4] were presented for comparison purposes. The transition probability matrix of a $K$-state SFM is calculated by expressing the EFRD $P\left(0^{m_{0}} / 1\right)$ 
as the sum of $K-1$ exponentials. In our case, the fitting of $P\left(0^{m_{0}} / 1\right)$ is achieved by using five exponentials. Note that no better performance can be obtained from SFMs with more than six states, which was also verified in [13]-[16]. As an example, we will only show the simulation results at a CIR of $9 \mathrm{~dB}$, which corresponds to a FER of 0.2882 . The chosen parameter vector for the corresponding deterministic process was $\boldsymbol{\Psi}=(9,10,0.09,0.0837,1.9046 \mathrm{~Hz}, 0.028 \mathrm{~s})$, which were calculated from the given quantities: $\mathcal{R}_{B}=0.7834$, $\mathcal{N}_{E B}=3489$, and $q_{s}=0.01$. Figs. 3-8 demonstrate the resulting GDs, EFRDs, ECDs, EBDs, BEPDs, and PECFs of both generative models and the descriptive model, respectively. The results for the EFBDs of the three models are not shown here since they are very close to each other. As expected, all these curves for the DPBGM have very excellent agreements, actually are nearly indistinguishable, with the target ones. However, relatively large deviations were found for the fittings to the desired ECD, EBD, BEPD, and BECF by using the SFM. This tells us that the SFM fails to model certain properties, especially the ECD, of the target error sequence. Both generative models require relatively long time in the simulation set-up phase, but the simulation run phase of the DPBGM is much faster than that of the SFM. For generating a packet error sequence of length $1.2 \times 10^{6}$, the DPBGM and SFM need about $0.63 \mathrm{~s}$ and $6.88 \mathrm{~s}$, respectively, for their simulation run phase.

\section{CONCLUSion}

In this paper, we have demonstrated a general procedure of developing a fast binary packet-level generative model with a properly parameterized and sampled deterministic process followed by a threshold detector and two parallel mappers. It is shown by simulation results that the proposed DPBGM has the attractive capability to reproduce accurately all the interested burst error statistics of the underlying descriptive model. Compared with a traditional Markov model, the suggested DPBGM is superior from both the accuracy and efficiency points of view. The developed DPBGM is very useful for the design and performance evaluation of media access control (MAC) layer [1], link control layer [6], [22], and higher layer wireless communication protocols [1].

\section{REFERENCES}

[1] M. Zorzi and R.R. Rao, "Perspectives on the impact of error statistics on protocols for wireless networks," IEEE Personal Commun., vol. 6, no. 10, pp. 32-40, Oct. 1999.

[2] P. M. Crespo, R. M. Pelz, and J. Cosmas, "Channel error profiles for DECT,' IEE Proceedings-Commun., vol. 141, no. 6, pp. 413-420, Dec. 1994.

[3] L. N. Kanal and A. R. K. Sastry, "Models for channels with memory and their applications to error control," Proc. of the IEEE, vol. 66, no. 7, pp. 724-744, July 1977.

[4] B. D. Fritchman, "A binary channel characterization using partitioned Markov chains," IEEE Trans. Information Theory, vol. 13, no. 2, pp. 221-227, Apr. 1967.

[5] A. Willig, "A new class of packet- and bit-level models for wireless channels," Proc. IEEE PIMRC'02, Lisbon, Portugal, Sept. 2002, pp. 2434-2440.

[6] A. Konrad, B. Y. Zhao, and A. D. Joseph, "A Markov-based channel model algorithm for wireless networks," Kluwer J. of Wireless Networks, vol. 9, no. 3, pp. 189-199, May 2003.

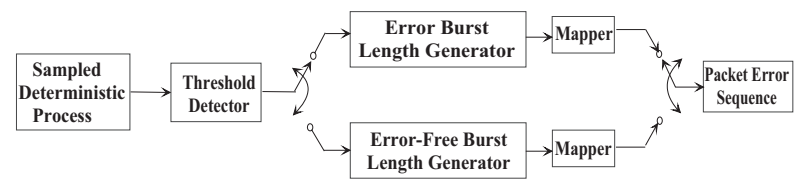

Fig. 1. The block diagram of the proposed DPBGM.

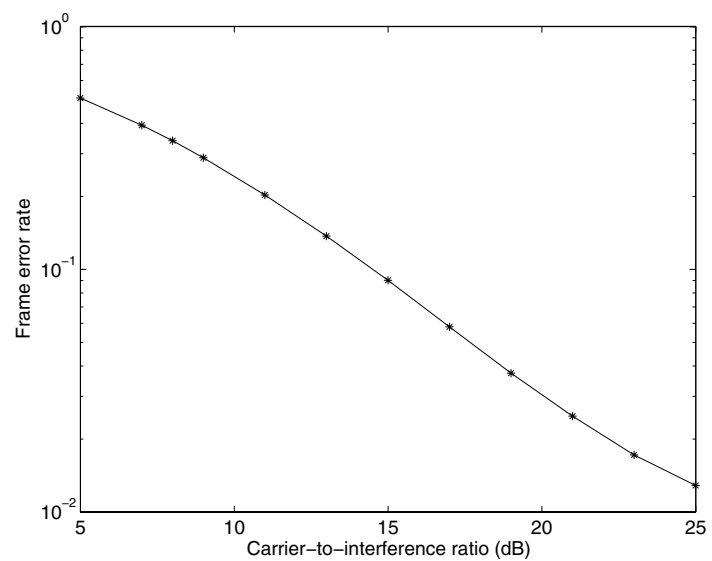

Fig. 2. The FER of the coded GPRS system with a TU channel.

[7] J. Aráuz and P. Krishnamurthy "Markov modeling of 802.11 channels," Proc. IEEE VTC'03-Fall, Florida, USA, Oct. 2003, pp. 771-775.

[8] N. Nefedov, "Discrete channel models for wireless data services," Proc. IEEE VTC'98, Ottawa, Canada, May 1998, pp. 683-687.

[9] W. Turin, Digital Transmission Systems: Performance Analysis and Modeling. New York: McGraw-Hill, 1999.

[10] E. Costamagna, L. Favalli, and P. Gamba, "Multipath channel modeling with chaotic attractors," Proc. of the IEEE, vol. 90, no. 5, pp. 842-859, May 2002.

[11] W. Zhu and J. G. Frias, "Stochastic context-free grammars and hidden Markov models for modeling of bursty channels," IEEE Trans. Veh. Technol., vol. 53, no. 3, pp. 666-676, May 2004.

[12] A. Köpke, A. Willig, and H. Karl, "Chaotic maps as parsimonious bit error models of wireless channels," Proc. IEEE INFOCOM'03, San Francisco, USA, Mar. 30-Apr. 3, 2003, pp. 513-523.

[13] C. X. Wang and M. Pätzold, "A generative deterministic model for digital mobile fading channels," IEEE Commun. Letters, vol. 8, no. 4, pp. 223-225, Apr. 2004.

[14] C. X. Wang and M. Pätzold, "Deterministic modeling and simulation of error sequences in digital mobile fading channels,' Proc. IEEE ICC'04, Paris, France, June 2003, pp. 3374-3378.

[15] C. X. Wang and M. Pätzold, "A new deterministic process based generative model for characterizing bursty error sequences," Proc. IEEE PIMRC'04, Barcelona, Spain, Sept. 2004, pp. 2134-2139.

[16] C. X. Wang, W. Xu, and M. Pätzold, "Error models for evaluating error control strategies in EGPRS systems," Proc. IEEE VTC'04-Fall, Los Angeles, USA, Sept. 2004.

[17] C. X. Wang, W. Xu, and M. Pätzold, "A novel generative model for the characterization of digital wireless channels with soft decision outputs,' Proc. NRS/FWCW'04, Oulu, Finland, Aug. 2004.

[18] 3GPP TS 45.005, "Radio transmission and reception (Rel. 4)," 2003.

[19] W. C. Jakes, Ed., Microwave Mobile Communications. New Jersey: IEEE Press, 1994.

[20] M. Pätzold, Mobile Fading Channels. New York: Wiley, 2002.

[21] C. X. Wang, M. Pätzold, and B. Itsarachai, "A deterministic frequency hopping Rayleigh fading channel simulator designed by using optimization techniques,' Proc. IEEE PIMRC'02, Lisbon, Portugal, Sept. 2002, pp. $478-483$.

[22] H. Jenkac, G. Liebl, T. Stockhammer, and W. Xu, "Flexible outer ReedSolomon coding on RLC layer for MBMS over GERAN," Proc. IEEE VTC'04-Spring, Milan, Italy, May 2004. 


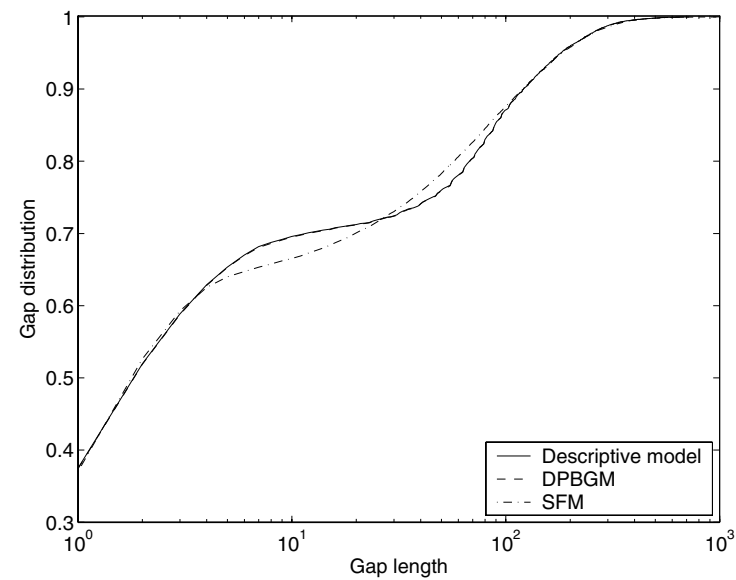

Fig. 3. The GDs of the generative models and the descriptive model.

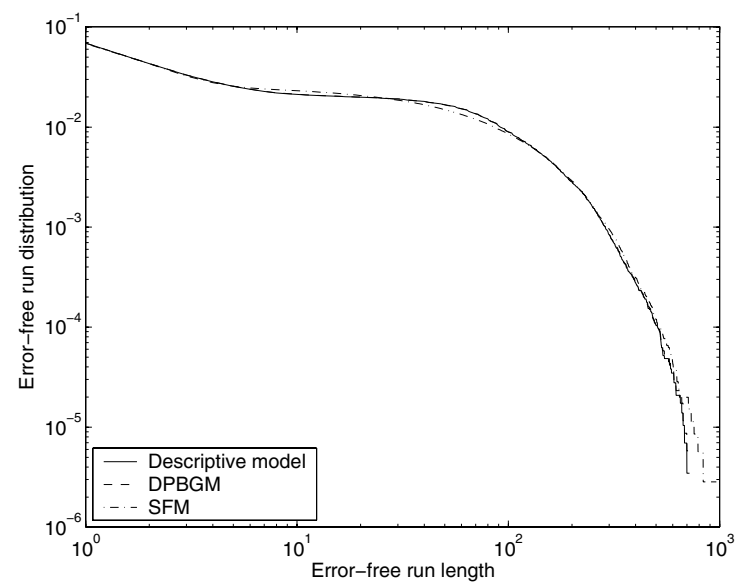

Fig. 4. The EFRDs of the generative models and the descriptive model.

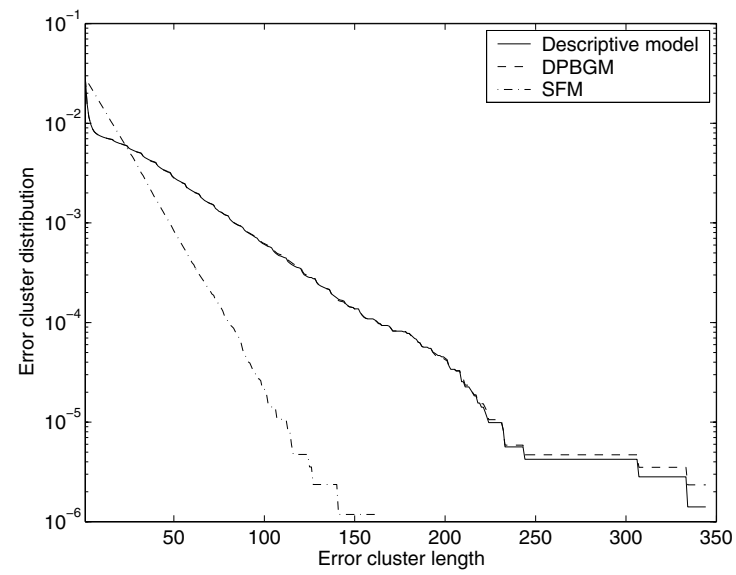

Fig. 5. The ECDs of the generative models and the descriptive model.

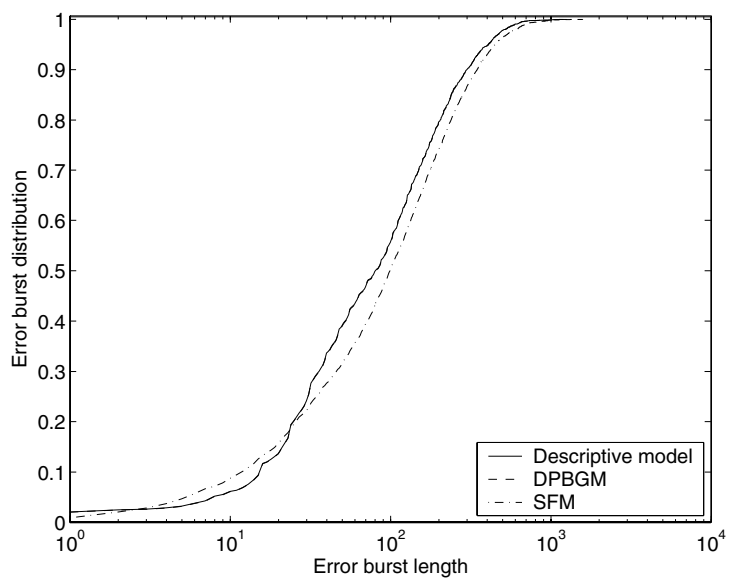

Fig. 6. The EBDs of the generative models and the descriptive model.

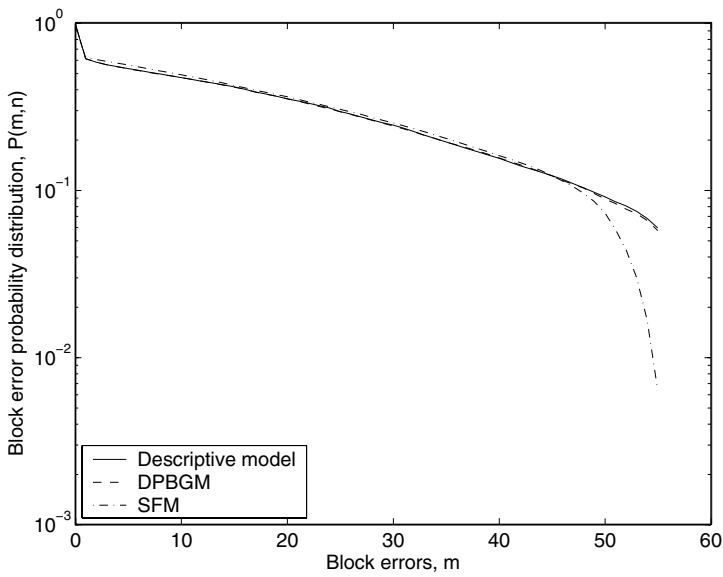

Fig. 7. The BEPDs of the generative models and the descriptive model.

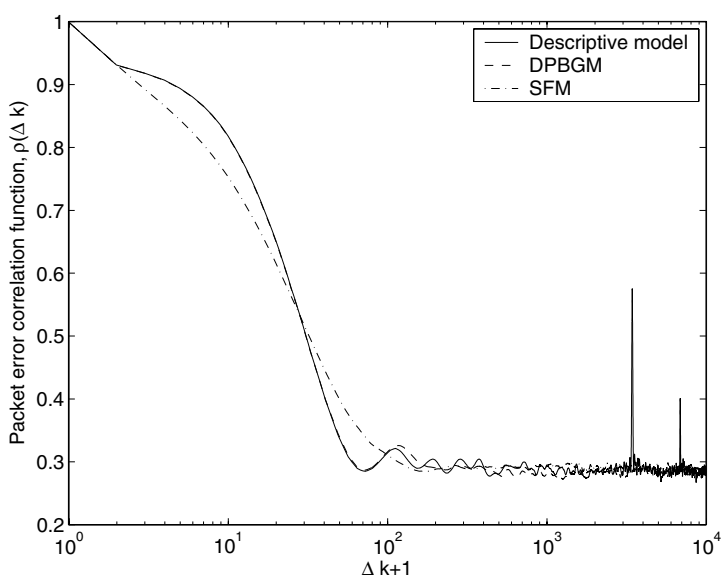

Fig. 8. The PECFs of the generative models and the descriptive model. 